\title{
Atividade Física Habitual e Qualidade de Vida de Mulheres na Meia-Idade
}

\author{
Habitual Physical Activity and Quality of Life of Middle-aged Women
}

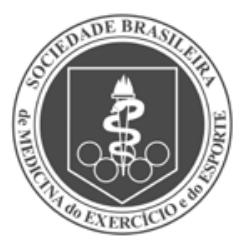

Artigo Original

Adriana Coutinho de Azevedo

Guimarães ${ }^{1}$

Fátima Baptista²

1. Departamento de Educação Física, Centro de Ciências da Saúde e do Esporte, Universidade do Estado de Santa Catarina, Brasil.

2. Laboratório de Exercício e Saúde, Faculdade de Motricidade Humana, Universidade Técnica de Lisboa, Portugal.

\section{Correspondência:}

Rua Bias Peixoto, 187, apto. 201 Itaguaçu || - 88085-480 - Florianópolis, SC, Brasil.

E-mails: nanacefid@hotmail.com; nanaguim@terra.com.br E-mail: fbaptista@fmh.utl.pt

\begin{abstract}
RESUMO
Objetivo: Analisar a relação entre a atividade física habitual (AF) e a qualidade de vida (QV) de mulheres na meia-idade. Métodos: Participaram do estudo 1.011 mulheres dos 45 aos 59 anos de idade, das quais 370 eram perimenopausadas e 641 pós-menopausadas, com índice de massa corporal (IMC) de $25 \pm 4 \mathrm{~kg} / \mathrm{m}^{2} \mathrm{e}$ escolaridade entre um e 13 anos. A QV foi avaliada pelo WHOQOL-bref da WHO e a AF através do International Physical Activity Questionnaire. Resultados: Após a divisão da amostra em três grupos, de acordo com a prática de AF total (A: < $30 \mathrm{~min} / \mathrm{dia}$; B: 30-60 min/dia; C: > $60 \mathrm{~min} / \mathrm{dia}$ ), a análise da variância ajustada para o IMC e menopausa (peri vs. pós), mostrou diferenças de QV entre os grupos $A$ e $B$ e o grupo $C$ no domínio físico, e entre o grupo A e os grupos B e C nos domínios psicológico, social e ambiental $(p \leq 0,001)$. Conclusão: A prática de $30 \mathrm{~min} /$ dia de AF de intensidade pelo menos moderada parece estar associada a efeitos mais favoráveis aos níveis psicológico, social e ambiental, mas parecem ser necessários pelo menos $60 \mathrm{~min} /$ dia para influenciar o domínio físico. Estas associações podem, no entanto, refletir o impacto da QV na quantidade total de AF. Assim, a prática de $30 \mathrm{~min} /$ dia pode ser mais condicionada pelos domínios psicológico, social e ambiental, enquanto os $60 \mathrm{~min} / \mathrm{dia}$ pelo domínio físico.
\end{abstract}

Palavras-chave: atividade física, mulheres, perimenopausa, pós-menopausa

\section{ABSTRACT}

Objective: To assess associations between of regular physical activity (PA) and quality of life (QL) in middle age women. Methods: 1011 female subjects, aged 45-59, took part in the research, considering 370 peri-menopausal and 641 post-menopausal, within the body mass index (BMI) $25 \pm 4 \mathrm{~kg} / \mathrm{m} 2$ and schooling average 1-13 years. The QL was evaluated by the WHOQOL, and the PA through the International Physical Activity Questionnaire. Results: After dividing the sample into three groups according to the practice of total PA (A: $<30 \mathrm{~min} /$ day; B: 30-60 min/day, C:> $60 \mathrm{~min} /$ day), analysis of variance adjusted for BMl and menopausal status (peri vs. postmenopause) showed differences in QL between groups $A, B$ and group $C$ in the physical domain and between group A and group B, C in psychological, social and environmental domains ( $p \leq 0.001)$. Conclusion: The accumulation of $30 \mathrm{~min} /$ day of total PA seems to be associated with more favorable effects on the psychological, social and environmental domains; however, it seems that at least $60 \mathrm{~min} /$ day are necessary to act on the physical domain. Nevertheless, such associations may reflect the impact of QL in the total amount of PA. Thus, the PA practice $30 \mathrm{~min} /$ day may be more conditioned by the psychological, social and environmental domains while the practice of $60 \mathrm{~min} /$ day by the physical domain.

Keywords: physical activity, women, perimenopause, postmenopause.

\section{INTRODUÇÃO}

A meia-idade compreende, aproximadamente, o período entre os 40 e os 59 anos $^{(1)}$, sendo considerada crítica particularmente nas mulheres devido às transformações determinadas biologicamente pela menopausa ${ }^{(2-4)}$. Estas transformações têm início entre os 39 e os 51 anos para 95\% das mulheres (idade média de início da transição para a perimenopausa - 46 anos) e são decorrentes do esgotamento dos folículos ovarianos (ou da remoção dos ovários, no caso da menopausa cirúrgica) ${ }^{(5)}$. Trata-se, geralmente, de um período de tempo acompanhado de sintomas de natureza física, alguns do quais específicos da menopausa como as ondas de calor e as dificuldades sexuais decorrentes da secura vaginal, e de outros sintomas mais gerais como a fadiga, as dores de cabeça e as insônias, que afetam 96\% das mulheres. Neste período, estão também presentes, em 63\% dos casos, sintomas de natureza psíquica como a depressão, a ansiedade, a irritabilidade e a diminuição da concentração( ${ }^{(6)}$. Estes sintomas têm uma duração média de cinco anos (duração para 95\% das mulheres dois a oito anos) ${ }^{(7)}$.

Para além da sintomatologia característica da transição perimenopausada, nesta idade podem igualmente surgir outras mudanças decorrentes de condicionantes psicossociais. A perda, o afastamento ou a separação de familiares e cônjuges, a independência e a saída de casa dos filhos, a gestão da carreira profissional e a estabilidade no mercado de trabalho, o envelhecimento em uma sociedade que valoriza a beleza e a juventude, assim como a manifestação de doenças crônicas, são situações que demandam ajustes emocionais ${ }^{(5,8)}$. No seu conjunto, estas alterações conduzem, frequentemente, a estados de depressão, crises de ansiedade, estresse crônico, além da baixa autoestima e autoimagem ${ }^{(9)}$

Apesar da potencial ocorrência de eventos negativos na meia-idade, esta fase do ciclo de vida encontra-se também associada a uma 
conquista da autonomia e do poder de tomada de decisão, à realização profissional, ao desenvolvimento e amadurecimento pessoal e ao alargamento de perspectivas para o futuro ${ }^{(10)}$. Para muitas mulheres a meia-idade constitui, no entanto, um período vulnerável acompanhado de uma diminuição da qualidade de vida nos domínios físico e psicológico comparativamente a homens da mesma idade ${ }^{(11)}$ ou a mulheres adultas jovens $^{(5,10,12-16)}$, e a uma diminuição da qualidade de vida nos domínios psicológico e social comparativamente a mulheres idosas ${ }^{(17)}$.

O exercício físico parece influenciar favoravelmente a qualidade de vida relacionada com a saúde, independentemente da idade e gênero ${ }^{(11,18-21)}$. Na meia-idade, programas de natureza aeróbia de intensidade pelo menos moderada, 30 minutos por dia, três a cinco dias por semana, com um a 12 meses de duração, revelaram benefícios na qualidade de vida em geral(22-24) e no domínio psicológico em particular, com uma diminuição da depressão e da ansiedade ${ }^{(25,26)}$. Estes trabalhos analisaram a influência do exercício físico na qualidade de vida em geral, existindo, no entanto, pouca informação sobre a especificidade dos seus efeitos nos diversos domínios da qualidade de vida relacionada com a saúde. Trata-se de um aspecto relevante, uma vez que diferentes quantidades e/ou intensidades de exercício físico podem influenciar diferentemente os diversos domínios da qualidade de vida. Por outro lado, a recomendação para a prática de atividade física no âmbito de saúde pública considera a atividade física acumulada semanalmente (150 min de intensidade pelo menos moderada) e não somente o exercício formal(27), tornando-se necessário analisar as relações entre esta recomendação e a qualidade de vida nas mulheres de meia-idade, uma vez que se trata de uma recomendação baseada em associações epidemiológicas entre a atividade física autorreportada e a saúde cardiovascular ou a saúde em geral(27-29) . Neste contexto, o principal objetivo deste estudo foi analisar a relação entre a quantidade e a intensidade da atividade física habitual e os diversos domínios da qualidade de vida relacionada com a saúde em mulheres de meia-idade.

\section{MÉTODOS}

\section{Sujeitos}

O cálculo do tamanho da amostra foi baseado na população de mulheres de 45 a 59 anos do município de Florianópolis, Santa Catarina, Brasil, de acordo com o último censo do Instituto Brasileiro de Geografia e Estatística ${ }^{(30)}$, com um erro aceitável de 5\%. Desta forma, foram incluídas no estudo 1.011 mulheres (para um tamanho mínimo da amostra de 1.000 mulheres), divididas em dois grupos de acordo com a função reprodutiva, nomeadamente, um grupo de mulheres perimenopausadas ( $n=370$ ) e outro grupo de mulheres pós-menopausadas $(n=641)$. As participantes foram convidadas a participar do estudo voluntariamente, através de instituições empregadoras ou prestadoras de serviços no nível da educação, saúde, desporto, religião e seguros.

Todas as mulheres foram informadas dos objetivos e procedimentos do estudo e assinaram o Termo de Consentimento Livre e Esclarecido (TCLE) conforme a Resolução CNS 196/96. Este estudo transversal decorreu entre dezembro de 2007 e março de 2009 após a sua aprovação pelo Comitê de Ética em Pesquisa da Universidade do Estado de Santa Catarina.

\section{PROCEDIMENTOS}

\section{Condição (perimenopausa vs. pós-menopausa)}

Cada participante foi questionada sobre há quanto tempo não menstruava. As mulheres com um a 11 meses de amenorreia foram consideradas na perimenopausa, e as mulheres com 12 meses ou mais de amenorreia foram consideradas na pós-menopausa ${ }^{(31)}$. Rela- tivamente à ocorrência da menopausa (natural ou cirúrgica), 18\% das mulheres tiveram menopausa cirúrgica, caracterizada pela retirada dos dois ovários. A idade da menopausa neste estudo foi calculada apenas com as mulheres que tiveram menopausa natural. A idade mínima de ocorrência da menopausa natural foi de 37 anos e a máxima de 57 anos, sendo a média etária de 45,4 \pm 3,5 anos.

\section{Atividade Física}

A atividade física foi avaliada através do questionário internacional de atividade física (IPAQ - versão curta), elaborado por pesquisadores de diversos países com suporte da Organização Mundial da Saúde (OMS), como parte de um estudo multicêntrico envolvendo 12 países. Cada país participante adaptou e validou o questionário de acordo com as características da respectiva população. No Brasil, este questionário foi validado pelo Centro de Estudos do Laboratório de Aptidão Física de São Caetano do Sul - CELAFISCS, que é o centro coordenador do IPAQ no Brasil(22). Na avaliação da atividade física habitual através do $I P A Q$, quantificou-se o número de vezes que um indivíduo realizou pelo menos 10 minutos contínuos de caminhada, de atividade física de intensidade moderada e de intensidade vigorosa na última semana, em diversos envolvimentos, nomeadamente, laboral, doméstico, lazer, recreativo e desportivo. Uma vez que à caminhada é geralmente atribuído um nível de exigência de 3,3METs, ou seja, um nível superior ao valor de corte para a atividade física moderada (3METs), a atividade física total, que resulta do somatório da caminhada, da atividade moderada e da atividade vigorosa, corresponde a uma atividade de intensidade pelo menos moderada.

\section{Qualidade de Vida}

A qualidade de vida foi avaliada através do questionário abreviado World Health Organization Quality of Life (WHOQOL), que se encontra adaptado para Português do Brasil( ${ }^{(33)}$. Trata-se de um questionário composto por 26 questões (das 100 originais), designadamente 24 questões que abrangem o domínio físico (dor física, energia, locomoção, atividades da vida diária, tratamento médico, trabalho), psicológico (sentimentos positivos, concentração, autoestima, autoimagem, sentimentos negativos, espiritualidade), social (relações pessoais, suporte social, atividade sexual) e meio ambiente (segurança física, habitação, recursos financeiros, serviço de saúde, informações, lazer, ambiente físico e transporte,) e mais duas questões gerais sobre qualidade de vida.

As questões, que se referem às duas últimas semanas, foram formuladas para uma escala de respostas do tipo Likert, expressas através de uma escala de intensidade (nada - extremamente), ou de capacidade (nada - completamente), ou de frequência (nunca - sempre), ou de avaliação (muito insatisfeito - muito satisfeito; muito ruim - muito bom), no qual os valores são computados em escores que variam de 0 (péssimo), 25 (regular), 50 (bom) 75 (muito bom) a 100\% (excelente).

A massa corporal, a estatura, o índice de massa corporal (peso $(\mathrm{kg})$ /altura $\left.(\mathrm{m})^{2}\right)$, os anos de escolaridade, o estado de saúde e a terapia de reposição hormonal foram avaliados por meio de um questionário autoaplicável.

\section{Análise dos dados}

A análise estatística foi efetuada através do SPSS - versão 16.0. A comparação das características das participantes (idade, índice de massa corporal e educação), da qualidade de vida e da atividade física entre mulheres peri e pós-menopausadas foi realizada através do teste $t$ para amostras independentes e a comparação da saúde e da reposição hormonal através do teste do Qui-quadrado. As associações entre a atividade física (caminhada, atividade física moderada, atividade física 
vigorosa, atividade física moderada mais vigorosa e atividade física total) e a qualidade de vida (domínio físico, domínio psicológico, domínio social e domínio ambiental) foram analisadas através de correlações parciais ajustadas para a condição (peri vs. pós-menopausa). A partir desta análise, procedeu-se à seleção da variável da atividade física meIhor correlacionada com os diversos domínios da qualidade de vida para dividir a amostra total em três grupos: A) < 30 min/dia; B) 30-60 min/dia; C) > 60 min/dia). A comparação entre estes três grupos foi efetuada através da ANCOVA ajustada para a condição (peri vs. pós-menopausa) e, em caso de diferenças significativas entre os grupos, através do teste post-hoc de Bonferroni. Devido ao tamanho da amostra, definiu-se o nível de significância para $p<0,01$.

\section{RESULTADOS}

A tabela 1 apresenta a caracterização da amostra, designadamente, a idade cronológica, da menarca, da menopausa, assim como o peso, a estatura, o número de anos de escolaridade, o número de doenças e a percentagem de participantes com terapia de substituição hormonal. As mulheres pós-menopausadas eram mais velhas, apresentando maior prevalência de doenças e fazendo maior uso da terapia hormonal de substituição. Nas demais variáveis não foram observadas diferenças entre os grupos. Considerando a amostra total, a idade média de ocorrência da menarca foi de 12,8 anos e a da menopausa de 45,4 anos (idade mínima de 37 anos e máxima de 57 anos). A escolaridade foi de 10,5 anos, mostrando que a maioria das mulheres estudou até o ensino médio. $O$ índice de massa corporal médio foi de $25,2 \mathrm{~kg} / \mathrm{m}^{2}$, revelando excesso de peso. Aproximadamente, $21 \%$ das mulheres faziam uso da terapia de reposição hormonal por período superior a cinco anos.

Tabela 1. Características das participantes do estudo (média \pm desvio padrão).

\begin{tabular}{|c|c|c|c|c|c|}
\hline & $\mathbf{n}$ & Total & Peri $M$ & Pós M & Valor de $p$ \\
\hline Idade cronológica (anos) & 1.011 & $49,5 \pm 5,1$ & $45,3 \pm 2,8$ & $52,0 \pm 4,5$ & $<0,001$ \\
\hline Idade da menarca (anos) & 986 & $12,8 \pm 1,7$ & $12,7 \pm 1,5$ & $12,9 \pm 1,8$ & 0,051 \\
\hline $\begin{array}{c}\text { Idade da menopausa } \\
\text { (anos) }\end{array}$ & 634 & - & - & $45,4 \pm 3,5$ & - \\
\hline Massa corporal $(\mathrm{kg})$ & 1.011 & $65,2 \pm 11,0$ & $65,3 \pm 10,8$ & $65,2 \pm 11,1$ & 0,812 \\
\hline Estatura (m) & 1.011 & $1,60 \pm 0,06$ & $1,61 \pm 0,06$ & $1,60 \pm 0,06$ & 0,032 \\
\hline $\begin{array}{l}\text { Índice de massa } \\
\text { corporal }\left(\mathrm{kg} / \mathrm{m}^{2}\right)\end{array}$ & 1.011 & $25,2 \pm 4,0$ & $25,1 \pm 3,9$ & $25,3 \pm 4,0$ & 0,431 \\
\hline Escolaridade (anos) & 1.010 & $10,5 \pm 3,1$ & $10,8 \pm 3,1$ & $10,4 \pm 3,1$ & 0,097 \\
\hline Doenças (\%) & 1.010 & & & & $<0,001$ \\
\hline Nenhuma doença & & 58,2 & 67,3 & 53,0 & \\
\hline Uma doença & & 26,1 & 22,7 & 28,2 & \\
\hline Duas doenças & & 10,2 & 6,4 & 12,4 & \\
\hline Três ou mais doenças & & 5,2 & 3,5 & 6,2 & \\
\hline Reposição hormonal (\%) & 1.005 & 21,1 & 10,9 & 26,9 & $<0,001$ \\
\hline
\end{tabular}

Peri $\mathrm{M}$ = perimenopausa: Pós $\mathrm{M}=$ pós-menopausa; $\mathrm{n}$ = número de participantes que respondeu às questões correspondentes a cada variável, à exceçăo da idade da menopausa em que o n expressa o número de participantes na menopausa.

Os resultados da qualidade de vida relacionada com a saúde variaram entre 59-61\% nos domínios físico e psicológico, e entre 67-69\% nos domínios ambiental e social, respectivamente, tendo em consideração a amostra total (Tabela 2). Não foram observadas diferenças na qualidade de vida entre os grupos de mulheres peri e pós-menopausadas.
Tabela 2. Comparação da qualidade de vida entre os grupos de mulheres peri e pós-menopausadas (média \pm desvio padrão).

\begin{tabular}{c|c|c|c|c|c}
\hline & $\mathbf{n}$ & Total & Peri M & Pós M & Valor de $\mathbf{p}$ \\
\hline Domínio físico (\%) & 999 & $59 \pm 14$ & $58 \pm 15$ & $59 \pm 13$ & 0,197 \\
\hline Domínio psicológico (\%) & 1.007 & $61 \pm 12$ & $61 \pm 13$ & $61 \pm 12$ & 0,841 \\
\hline Domínio social (\%) & 1.009 & $69 \pm 20$ & $68 \pm 21$ & $70 \pm 20$ & 0,226 \\
\hline Domínio ambiental (\%) & 1.011 & $67 \pm 16$ & $66 \pm 16$ & $70 \pm 16$ & 0,414 \\
\hline
\end{tabular}

Peri $\mathrm{M}$ = perimenopausa; Pós $\mathrm{M}$ = pós-menopausa; $\mathrm{n}$ = número de participantes que respondeu às questõe correspondentes a cada variável.

A tabela 3 apresenta os resultados referentes à atividade física discriminados pelo tempo despendido na caminhada, em atividade física de intensidade moderada, de intensidade vigorosa, de intensidade moderada e vigorosa e de atividade física total. Não foram observadas diferenças de atividade física entre os grupos, à exceção da atividade vigorosa em que as mulheres pós-menopausadas reportaram valores diários superiores às mulheres perimenopausadas. De uma forma geral, as participantes desta pesquisa adotaram a caminhada como forma de atividade física, caminhando em média 48 minutos por dia. Relativamente à atividade física total, constatou-se que os valores reportados se situam muito acima do que é recomendado para a população adulta, ou seja, uma média de 90 minutos por dia (caminhada + moderada + vigorosa). Uma vez que as diferenças da qualidade de vida e da atividade física entre as mulheres peri e pósmenopausadas foram praticamente inexistentes, a análise das associações entre a qualidade de vida e a atividade física foi efetuada tendo em consideração a amostra total, ajustando-se, todavia, esta análise para a condição menopausal (Tabela 4). De um modo geral, os resultados revelaram associações positivas entre os domínios da qualidade de vida e a atividade física de intensidade moderada, de intensidade vigorosa, de intensidade moderada e vigorosa e a atividade física total $(p<0,01)$. Não foram observadas associações entre a qualidade de vida e a caminhada.

Tabela 3. Comparação da atividade física entre os grupos de mulheres peri e pósmenopausadas (média \pm desvio padrão)

\begin{tabular}{c|c|c|c|c|c}
\hline & $\mathbf{n}$ & Total & Peri M & Pós M & Valor de p \\
\hline Caminhada (min/d) & 994 & $48 \pm 43$ & $46 \pm 40$ & $50 \pm 45$ & 0,284 \\
\hline AF moderada (min/d) & 995 & $28 \pm 40$ & $29 \pm 40$ & $27 \pm 39$ & 0,420 \\
\hline AF vigorosa (min/d) & 995 & $15 \pm 32$ & $12 \pm 30$ & $16 \pm 34$ & 0,009 \\
\hline AF Mod + Vig (min/d) & 995 & $43 \pm 56$ & $41 \pm 52$ & $44 \pm 58$ & 0,941 \\
\hline AF total (min/d) & 994 & $90 \pm 75$ & $87 \pm 68$ & $93 \pm 78$ & 0,508 \\
\hline
\end{tabular}

Peri $\mathrm{M}$ = perimenopausa; Pós $\mathrm{M}$ = pós-menopausa; $\mathrm{AF}$ = atividade física; Mod + Vig = moderada + vigorosa; $n=$ número de participantes que respondeu às questões correspondentes a cada variável.

Tabela 4. Correlações parciais entre a atividade física e a qualidade de vida de mulheres na meia-idade.

\begin{tabular}{c|c|c|c|c|c}
\hline & Caminhada & AF Mod & AF Vig & AF Mod+Vig & AF Total \\
\hline Domínio físico (\%) & 0,067 & $0,086^{*}$ & $0,109^{*}$ & $0,126^{*}$ & $0,132^{*}$ \\
\hline Domínio psicológico (\%) & 0,079 & $0,078^{*}$ & $0,086^{*}$ & $0,107^{*}$ & $0,124^{*}$ \\
\hline Domínio social (\%) & 0,052 & $0,083^{*}$ & $0,096^{*}$ & $0,116^{*}$ & $0,116^{*}$ \\
\hline Domínio ambiental (\%) & 0,040 & $0,105^{*}$ & 0,051 & $0,105^{*}$ & $0,101^{*}$ \\
\hline
\end{tabular}

AF = atividade física; Mod = moderada; Vig = vigorosa; ${ }^{*} p<0,01$

Correlaçōes parciais ajustadas para a condição menopausal (peri vs. pós-menopausa). 
Quando a amostra foi dividida em três grupos de acordo com a prática de atividade física total habitual (A: < 30 min/dia; B: 30-60 min/ dia; C: > $60 \mathrm{~min} /$ dia) ajustada para a condição menopausal (peri vs. pós-menopausa), verificou-se um maior tempo de atividade física total habitual nas mulheres que possuíam os escores de qualidade de vida mais elevados nos domínios psicológico, social e ambiental comparativamente às mulheres que realizavam menos atividade física (<30 min/ dia) ( $p \leq 0,001$ ) (Tabela 5). No domínio físico, as mulheres com uma prática diária de atividade física total superior a 60 minutos demonstraram valores mais elevados da qualidade de vida comparativamente às que não realizavam esta quantidade $(p<0,001)$.

Tabela 5. Comparação da qualidade de vida de acordo com a prática de atividade física total de mulheres na meia-idade.

\begin{tabular}{c|c|c|c|c|c}
\hline & A & B & C & Valor de $\mathbf{p}$ & Post-hoc \\
\hline & $<\mathbf{3 0 ~} \mathbf{m i n} / \mathbf{d}$ & $\mathbf{3 0 - 6 0} \mathbf{~ m i n} / \mathbf{d}$ & $>\mathbf{6 0} \mathbf{~ m i n} / \mathbf{d}$ & & \\
\hline Domínio físico (\%) & $57 \pm 0,7$ & $58 \pm 0,7$ & $60 \pm 0,5$ & $<0,001$ & $\mathrm{~A}, \mathrm{~B}<\mathrm{C}$ \\
\hline $\begin{array}{c}\text { Domínio } \\
\text { psicológico (\%) }\end{array}$ & $58 \pm 0,7$ & $61 \pm 0,7$ & $63 \pm 0,5$ & $<0,001$ & $\mathrm{~A}<\mathrm{B}, \mathrm{C}$ \\
\hline Domínio social (\%) & $65 \pm 1,3$ & $69 \pm 1,2$ & $72 \pm 0,9$ & 0,001 & $\mathrm{~A}<\mathrm{B}, \mathrm{C}$ \\
\hline $\begin{array}{c}\text { Domínio } \\
\text { ambiental (\%) }\end{array}$ & $63 \pm 1,0$ & $67 \pm 0,9$ & $68 \pm 0,7$ & 0,001 & $\mathrm{~A}<\mathrm{B}, \mathrm{C}$ \\
\hline
\end{tabular}

ANCOVA ajustada para a condição menopausal (peri vs. pós-menopausa).

\section{DISCUSSÃO}

O principal objetivo deste estudo foi analisar as relações entre a intensidade e a quantidade da atividade física habitual e os diversos domínios da qualidade de vida relacionada com a saúde em mulheres de meia-idade. Os resultados indicam que a prática de 30 min/ dia de atividade física total pode estar associada a características mais favoráveis ao nível psicológico, social e ambiental, mas parecem ser necessários pelo menos $60 \mathrm{~min} /$ dia para efeitos adicionais a nível físico.

A atividade física total quantificada pelo IPAQ considera unicamente a atividade física com um nível de exigência mínimo de 3,3METs (caminhada) e, consequentemente, a atividade física total diz respeito à atividade de intensidade pelo menos moderada. Assim, poder-se-á afirmar que a recomendação de saúde pública para a atividade física poderá não ser suficiente para influenciar o domínio físico da qualidade de vida. Estes resultados podem, no entanto, refletir a influência diferenciada de diversos domínios da qualidade de vida na atividade física habitual. Neste caso, a prática de uma menor quantidade de atividade física de intensidade pelo menos moderada, especificamente $30 \mathrm{~min} /$ dia, parece ser mais condicionada por fatores de natureza psicológica, social ou ambiental, enquanto uma acumulação superior a $60 \mathrm{~min} /$ dia parece ser mais determinada por fatores de natureza física. Entre estes fatores, as dores, a fadiga e a insônia (domínio físico), a autoestima e a autoimagem (domínio psicológico), assim como os recursos financeiros, o ambiente físico e o transporte (domínio ambiental) têm sido apontados como sendo os mais determinantes de uma menor ou maior prática de atividade física(34,35).

Embora diversos trabalhos transversais ou de intervenção descrevam uma associação/influência da atividade/exercício físico na qualidade de vida nos domínios físico e psicológico, a sua quantidade e intensidade não têm sido objeto de avaliação(22,23,25,36,37). Por outro lado, investigações que relacionem a atividade física e a qualidade de vida nos domínios social e ambiental são praticamente inexistentes, à exceção de Mirzaiinjmabadi et al.(38), que observaram melhoria da qualidade de vida em nível sexual (domínio social) em mulheres de 45-60 anos.

Tal como em outros estudos, a atividade física total parece contribuir para a qualidade de vida relacionada com a saúde em mulheres de meia-idade ${ }^{(26,39)}$, apesar da variação de qualquer domínio da qualidade de vida explicada pela atividade física ser inferior a 2\%. Mesmo assim, níveis suficientes de atividade física parecem proporcionar um menor número de dias não saudáveis ${ }^{(39)}$.

As participantes deste estudo demonstraram uma boa qualidade de vida, com escores médios ( 60-70\%) semelhantes aos observados em outros trabalhos com o mesmo tipo de população(14,16,25,40-42). Não foram, todavia, tal como em outros estudos, observadas diferenças de qualidade de vida entre mulheres peri e pós-menopausadas ${ }^{(13,14,43)}$. Nestes estudos, as principais diferenças relatadas estavam associadas às ondas de calor, insônia e dores musculares ${ }^{(14)}$, à inatividade física ${ }^{(43)}$ e ao estresse(13).

No presente estudo, a prevalência das mulheres suficientemente ativas em uma perspectiva de saúde foi de 63\% nas mulheres perimenopausadas e de $65 \%$ nas mulheres pós-menopausadas. Trata-se de valores de prevalência semelhantes aos observados no Brasil em 2003, em mulheres dos 40-65 anos (62\%) através do IPAQ em uma amostra de 511 mulheres dos 18 aos 65 anos de São Paulo(44). Além disso, observou-se uma prática habitual de atividade física total de 90 minutos por dia, ou seja, o triplo da recomendação para pessoas adultas (30 min/dia), não existindo diferenças de acordo com a fase do ciclo biológico (peri vs. pós-menopausa). A quantidade elevada de atividade física reportada por estas mulheres pode ser justificada pelo fato de o IPAQ avaliar a atividade física em diversos contextos, incluindo o profissional, o doméstico e o de transporte, e não se restringir apenas às atividades realizadas no lazer. Em países em desenvolvimento como o Brasil, as atividades relacionadas com a ocupação e os transportes ${ }^{(45)}$, assim como as tarefas domésticas, representam uma parte significativa da atividade total das mulheres de meia-idade. Na realidade, na idade adulta, parece que o período de vida em que se verifica uma maior prevalência da atividade física das mulheres se situa entre os 40 e os 49 anos $^{(46)}$, apesar de a prática formal de exercício poder ser superior nas mulheres pós-menopausadas do que nas pré-menopausadas ${ }^{(25,47)}$.

\section{CONCLUSÃO}

Concluindo, a prática de $30 \mathrm{~min} /$ dia de atividade física de intensidade pelo menos moderada pode estar associada a efeitos mais favoráveis ao nível psicológico, social e ambiental, mas parecem ser necessários pelo menos $60 \mathrm{~min} /$ dia para efeitos significativos a nível físico. Estas associações podem, no entanto, refletir o impacto da qualidade de vida na quantidade total de atividade física. Assim, a prática de $30 \mathrm{~min} /$ dia parece ser mais condicionada pelos domínios psicológico, social e ambiental, enquanto os $60 \mathrm{~min} /$ dia pelo domínio físico.

Todos os autores declararam não haver qualquer potencial conflito de interesses referente a este artigo.

\section{REFERÊNCIAS}

1. Andrews M. The seductiveness of agelessness. Ageing Society 2000;19:301-18.

2. Soares $C N$, Cohen LS. Mood disturbance, perimenopause, and hormone variability. São Paulo Med J 2001;119:78-83.

3. Dennerstein L, Guthrie JR, Clarck M, Lehert P, Henderson VW. A population-base study of depressed mood in middle-aged, autralian-born women. Menopause 2004;11-563-8.

4. Blumel JE, Castelo-Branco C, Binfa L, Gramegna G, Tacla X, Aracena B, et al. Quality of life after the menopause a population study. Maturitas 2000;34:17-23.

5. Dennerstein $L$, Lehert $P$, Guthrie J. The effects of the menopausal transition and biopsychossocial factors on well-being. Arch Wom Ment Health 2002;5:15-22.

6. Matthews KA, Bromberger JT. Does the menopausal transition affect health related quality of life? Am J Med 2005;8:25-36. 
7. Speroff L, Fritz MA. Clinical gynecologic endocrinology and infertility. Edition 7, Lippincott, Williams \&Wilkins, 2005.

8. Olofsson AS, Collins A. Psychosocial factors, attitude towards menopause and symptoms in Swedish peri-menopausa women. Climacteric 2000;3:33-42.

9. Khademi S, Cooke MS. Comparing the attitudes of urban and rural Iranian women toward menopause. Maturitas 2003:46:113-21.

10. Mori M, Coelho V. Women in body and soul: biopsychossocial factors in menopause. Psicol Reflex Crit 2004;17:177-87.

11. Sguizzatto GT, Garcez-Leme LE, Casimiro L. Evaluation of the quality of life among elderly female athletes. São Paulo Med J 2006;124:5-15.

12. Zapantis G, Santoro N. The menopausal transition: characteristics and management. Best Prac Res Clin Endocrinol Metab 2003;17:33-52

13. Avis NE, Assmann SF, Kravitz HM, Ganz PA, Ory M. Quality of life in diverse groups of midlife women: assessing the influence of menopause, health status and psychosocial and demographic factors. Qual life Res 2004;13:933-46.

14. Waidyasekera $\mathrm{H}$, Wijewardena $\mathrm{K}$, Lindmark G, Naessen T. Menopausal symptoms and quality of life during the menopausal transition in Sri Lankan women. Menopause 2009;16:164-70.

15. Blumel JE, Cruz MN, Aparício NJ. La transicion menopausica: fisiopatologia clinica y tratamiento Menopausal transition: physiopathology, clinical and treatment. Rev Medicina (B.Aires) 2002;62:57-65.

16. Fuh J, Wang $S$, Lee $S$, Juang K. Quality of life and menopausal transition for middle-age women on Kinneen island. Qual life Res 2003;12:53-61.

17. Nappi RE, Lachowsky M. Menopause and sexuality: Prevalence of symptoms and impact on quality of life. Maturitas 2009;63:138-41.

18. Spirduso WW, Cronin DL. Exercise dose-response effects on quality of life and independent living in older adults. Med Sci Sports Exerc 2001;33:598-608.

19. King AC, Pruitt LA, Phillips W, Oka R, Rodenburg A, Haskell WL. Comparative effects of two physical activity programs on measured and perceived physical functioning and other health-related quality of life outcomes in older adults. J Gerontol A Biol Sci Med Sci 2000;55:74-83.

20. Dorn J, Vena J, Jonh EM, Horn-Ross JL, Koo J. Lifetime physical activity and breast cancer risk in pre an postmenopausal women. Med Sci Sports Exerc 2003;35:278-85.

21. Wolin KY, Colditz GA, Glynn R, Lee I-Min, Kawachi I. Long-term physical activity and health-related quality of life in middle aged women. Med Sci Sports Exerc 2005;37:45-55.

22. Teoman N, Özcan A, Acar B. The effect of exercise on physical fitness and quality of life in postmenopausal women. Maturitas 2004;4:71-7.

23. Moriyama CK, Oneda B, Bernardo FR, Cardoso CG Jr, Forjaz CLM, Abrahao SB,et al. A randomized, placebo-controlled trial of the effects of physical exercises and estrogen therapy on health-related quality of life in postmenopausal women. Menopause 2008;15:613-8.

24. Villaverde-Gutierrez C, Araujo E, Cruz F, Roa JM, Barbosa W, Ruiz-Villaverde G. Quality of life of rural menopausal women in response to a customized exercise programme. J Advanc Nurs. 2006;54:11-9.

25. Asbury EA, Chandrruangphen $P$, Collins P. The importance of continued exercise participation in quality of life psychological well-being in previously inactive postmenopausal women: a pilot study. Menopause 2006;13:561-7.

26. Daley A, MacArthur C, Mutrie N, Stokes-Lampard H. Exercise for vasomotor menopausal symptoms. Cochrane Database Syst Rev 2007;17:CD006108.
27. WHO. Steps to Health: A European Frame Work to Promote Physical Activity for Health. 2007 Copenhaya: World Health Organization - Regional Office for Europe.

28. Morris JN, Heady JA, Raffle PAB, Roberts CG, Parks JW. Coronary heart-disease and physical activity of work. Lancet 1953;265:1053-7.

29. Paffenbarger RS, Blair SN, Kohl HW, Clark DG, Cooper KH, Gibbons LW. Physical activity, all-cause mortality, and longevity of college alumni. N Engl J Med 1986;314:605-13.

30. IBGE - Instituto Brasileiro de Geografia e Estatística. Projeção da população do Brasil/censo 2000 Web site [internet]. [citado em 15 fev 2007]. Available from: http//www.ibge.gov.br.

31. Burger HG. The menopausal transition. Bailliere`s Clin Obstet Gynecol 1996;10:347-58.

32. Pardini $R$, Matsudo $S$, Matsudo TAV, Andrade E, Braggion G, Andrade $D$, Oliveira L, Figueira Júnior $A$, Raso V. Validation of the international physical activity questionnaire (IPAQ): pilot study in brazilian young adults. Med Sci Sports Exerc 1997;29:S5-9.

33. Fleck MPA, Lima AFBS, Polanczyk CA, Trentini CM, Bandeira D, Patrick DL, et al. Aplicação da versão em português do instrumento Whoqol abreviado. Rev Saúde Pública 1999;34:178-83.

34. Seefeld V, Malina RM, Clarck MA. Factors affecting levels of physical activity in adults. Sports Med 2002;32:143-68

35. De Lorenzi DRS, Baracat EC, Saciloto B, Padilha Júnior I. Fatores associados a qualidade de vida após a menopausa. Rev Assoc Med Bras 2006;52:312-7.

36. Elavsky S, McAuley E. Exercise and self-esteem in menopausal women: a randomized controlled trial involving walking and yoga. Am J Health Promot 2007;22:83-92.

37. Elavsky S, McAuley E. Physical activity and mental health outcomes during menopause: a randomized controlled trial. Ann Behav Med 2007;33:132-42.

38. Mirzaiinjmabadi KM, Anderson D, Barnes M. The relationship between exercise, body mass index and menopausal symptoms in midlife Australian women. Int J Nurs Pract 2006;12:28-34.

39. Brown DW, Brown DR, Heath GW, Balluz L, Giles WH, Ford ES, et al. Associations between physical activity dose and health-related quality of life. Med Sci Sports Exerc 2004;36:809-96.

40. Rukwong PMNS, Chirawatkul S, Markovic M. Quality of life perceptions of middle-aged women living with a disability in muang district, Khon Kaen, Thailand: Whogol perspective. J Med Assoc Thai 2007;90:1640-6

41. Galvão LLL, Farias MCS, Azevedo PRM, Vilar MJP, Azevedo GD. Prevalência de transtornos mentais comuns e avaliação da qualidade de vida no climatério. Rev Assoc Med Bras 2007;53:415-20.

42. Hsu MC, Wang TS, Liu YP, Liu CF. Effects of baduanjin exercise on oxidative stress and antioxidant status and improving quality of life among middle-aged women. Am J Chin Med 2008;36:815-26.

43. Misha G, Kuh D. Perceived change in quality of life during the menopause. Soc Sci Med 2006;62:93-102.

44. Bauman, A., F. Bull, T. Chey, C.L. Craig, B.E. Ainsworth, J.F. Sallis, H.R. Bowles, M. Hagstromer, M. Sjostrom, and M. Pratt. The International Prevalence Study on Physical Activity: results from 20 countries. Int Behav Nutr Phys Act 2009;6:21-32.

45. Hallal PC, Victoria CG, Wells JCK, Lima RC. Physical inactivity: prevalence and associated variables in brazilian adults. Med Sci Sports Exerc 2003;35:1894-900.

46. Instituto do Desporto de Portugal. Observatório Nacional da Actividade Física - Livro Verde da Actividade Física. IDP, Lisboa, 2010.

47. Asikainen TM, Kukkinen-Harjula K, Miilunpalo S. Exercise for helath for early postmenopausal women Sports Med 2004;34:753-78. 